\title{
Open-Loop Control for Focusing of Neutral Particles by Modulated Optical Field
}

\author{
S. BORISENOK ${ }^{a, b, *}$ AND N. IQBAL ${ }^{a}$ \\ ${ }^{a}$ Abdus Salam School of Mathematical Sciences, GC University \\ 35-C/2, Gulberg 3, Lahore, Pakistan \\ ${ }^{b}$ Dept. of Physics, Herzen State Pedagogical University \\ 48 Moika River, 191186 St. Petersburg, Russia \\ (Received February 14, 2008; revised version March 29, 2008; \\ in final form June 11, 2008)
}

We propose open-loop control model of focusing cooled quantum particles in 1D and 2D modulated optical field. Applying Kapitza's procedure of averaging, we demonstrate that the type of attractor in the fixed points can be controlled by changing the parameters of control optical field. We support our conclusions by simulation for harmonic control.

PACS numbers: 42.50.-p, 02.30.Yy

\section{Introduction}

The dynamics of neutral quantum particles in standing laser wave field is a subject of great interest, stimulated by the focusing problems in nanolithography with cooled atoms. The main task is to obtain different distributions of cooled atoms along the standing optical wave, for instance, to form very narrow periodical structure with a spatial period that is much less than the wavelength of the optical radiation. The effective coherent splitting has been achieved by scattering of atom wave packets by additional optical field with modulated intensity [1-3].

Dynamics of a neutral quantum particle (cooled atom), moving along $x$, in the field of the perpendicular standing optical wave (along $y$ ) can be described in the terms of classical pendulum with a friction [4]:

$$
\ddot{y}+\beta \dot{y}+\omega^{2} \sin y=0,
$$

where overdots stand for time derivatives, the constant $\beta$ plays the role of the

*corresponding author; e-mail: nayyersms3@gmail.com 
"friction coefficient" for atoms, $\omega$ is an oscillation frequency of the particles in the single potential minimum, created by the standing optical field, and the coordinate $y$ is dimensionless, scaled by the wave number. In Eq. (1) the diffusion term is neglected, i.e. we consider the time of the interaction between the atoms and the optical field to be short.

We investigate the possibility to increase the focusing effect without changing the length of the optical field. We apply a control laser field, that leads to the additional term in dynamic equation for the single particle motion

$$
\ddot{y}+\beta \dot{y}+\omega^{2}(1+u) \sin y=0 .
$$

Here $u$ is the control action [5], which may depend on current position and velocity of the atoms. In this case the closed-loop (feedback) algorithm should be applied [4]. Nevertheless, it can be very difficult to organize the fast measurement of the atomic distribution along $y$-direction, and the feedback scheme looks over-sophisticated for practical realization of focusing. For this reason we will concentrate below on open-loop (feedforward) algorithms of control. It means that we suppose the signal $u(t)$ to be dependent on time only.

The goal of this article is to formulate the simple mathematical model for the process of $1 \mathrm{D}$ and $2 \mathrm{D}$ focusing in the frame of open-loop control. We investigate the opportunity to fabricate narrow spatial periodical structures by application of simple form of fast optical modulation. In Sect. 2 we discussed our approach in detail for $1 \mathrm{D}$ case, and then in Sect. 3 we present some features of 2-dimensional focusing. In Appendix we support our conclusions by numerical simulations for harmonic control.

\section{Mathematical model of one-dimensional focusing}

To apply efficiently open-loop algorithm for the fabrication of narrow spatial structures we will use the idea of the Kapitza averaging, first proposed in [6] and described in detail in [7]. Let us consider the case of $1 \mathrm{D}$ focusing by fast oscillating control $u(t)$. For simplicity we will discuss here the harmonic case

$$
u(t)=A \cos (\nu t+\varphi)
$$

with constant $A, \nu, \varphi$. We demand: $\nu \gg \omega$.

Following $[6,7]$, for the sufficiently large frequency $\nu$ we can present the coordinate $y$ as a superposition of its "smooth" part $Y$ and the fast changing component $\eta$ :

$$
y=Y+\eta
$$

the last one comes from the control perturbation. We define $\eta$ by

$$
\ddot{\eta}+\beta \dot{\eta}+\omega^{2} u(t) \sin Y=0 .
$$

Here $Y$ is quasi-integral of motion. Because the control is fast, we will omit the friction coefficient, supposing that the $\beta$-decay is slow to compare with the control period $2 \pi / \nu$. Then Eq. (4) becomes

$$
\ddot{\eta}+\omega^{2} A \cos (\nu t+\varphi) \sin Y=0
$$


with the solution

$$
\eta=A \epsilon \sin Y \cos (\nu t+\varphi),
$$

where $\epsilon=\omega^{2} / \nu^{2}$ is a small parameter. Now we can expand $\sin Y$ in the Taylor series, keeping the second order of $\eta$ :

$$
\sin Y=\sin Y+\eta \cos Y-\frac{1}{2} \eta^{2} \sin Y
$$

and substitute it into equation

$$
\ddot{Y}+\ddot{\eta}+\beta(\dot{Y}+\dot{\eta})+\omega^{2}(1+u) \sin (Y+\eta)=0
$$

taking into consideration the definition (4). The key step is the Kapitza averaging

$$
\langle\ldots\rangle=\frac{1}{\tau} \int_{0}^{\tau} \ldots \mathrm{d} t
$$

with the properties

$$
\langle Y\rangle=Y, \quad\langle\eta\rangle=0,
$$

such that, for example,

$$
\langle\eta \cos Y\rangle=\cos Y\langle\eta\rangle \equiv 0,
$$

etc. Then the averaged Eq. (6) can be written as

$$
\ddot{Y}+\beta \dot{Y}+\omega^{2}\left(1-\frac{1}{2}\left\langle\eta^{2}\right\rangle\right) \sin Y+\omega^{2}\left(\langle u \eta\rangle \cos Y-\frac{1}{2}\left\langle u \eta^{2}\right\rangle \sin Y\right)=0,
$$

where

$$
\left\langle\eta^{2}\right\rangle=\frac{1}{2} A^{2} \epsilon^{2} \sin ^{2} Y, \quad\langle u \eta\rangle=\frac{1}{2} A^{2} \epsilon \sin Y
$$

Finally, saving the terms of the zero and first order with respect to the small parameter $\epsilon$, we get a closed equation for the "slow" coordinate $Y$ of the particle

$$
\ddot{Y}+\beta \dot{Y}+\omega^{2} \sin Y+\frac{1}{2} \omega^{2} A^{2} \epsilon \sin Y \cos Y=0
$$

Thus, the correction of the smooth movement $Y$ by fast control leads to the famous Kapitza effect of the design for stability points of the dynamical system. It is sufficient if the coefficient $A^{2} \epsilon$ is not small, i.e. if the amplitude $A$ of the control is large (because $\epsilon$ is a small parameter by definition).

To check it we calculate the Lyapunov spectra [8] for the system (8). The fixed points of this system on the phase plane $(Y, \dot{Y})$ are $(0,0),(\pi, 0)$ and $\left(\arccos \left(-2 / A^{2} \epsilon\right), 0\right)$. We remind that here, despite the looking of Eq. (2) like a mechanical pendulum equation, our phase space is not cylindric, and every fixed point mentioned above corresponds to the infinite set, like: $(\pi, 0)$ stands for $(\pi+2 \pi n, 0)$, where $n$ is integer, and so on. We are interested in the Lyapunov spectra with the signs $\{-,-\}$ to reproduce a focusing effect.

At the fixed point $(0,0)$ the Lyapunov spectrum is always $\{-,-\}$. It means that the focusing around these points always presents in our periodical structure, 
and it reproduces the period of the standing wavelength. At the fixed point $(\pi, 0)$, corresponding to the "inverse position" of the "pendulum", the Lyapunov spectrum is $\{-,-\}$ if

$$
\frac{2}{A^{2}}<\epsilon \ll 1
$$

and at the non-trivial point $\left(\arccos \left(-2 / A^{2} \epsilon\right), 0\right)$ the spectrum $\{-,-\}$ is impossible.

In general, such a structure corresponds to the famous case of vertically driven Kapitza pendulum. Its inverse position becomes stable under the condition (9). Thus, we can switch on and off this periodicity of focusing, changing the parameter $A$.

\section{Some features of 2-dimensional case}

Equation (2) can be easily generalized for the 2D case, when two optical fields interact with the atomic beam in perpendicular directions to the beam and to each other

$$
\begin{aligned}
& \frac{\mathrm{d}^{2} y}{\mathrm{~d} t^{2}}+\beta \frac{\mathrm{d} y}{\mathrm{~d} t}+\omega_{1}^{2}\left[1+u_{1}(t)\right] \sin y=0 \\
& \frac{\mathrm{d}^{2} z}{\mathrm{~d} t^{2}}+\beta \frac{\mathrm{d} z}{\mathrm{~d} t}+\omega_{2}^{2}\left[1+u_{2}(t)\right] \sin z=0
\end{aligned}
$$

This system is shown in Fig. 1. Here $\beta, \omega_{1}, \omega_{2}$ are positive coefficients, and $u_{1}$ and $u_{2}$ correspond to two perpendicular control fields along $y$ and $z$ correspondingly.

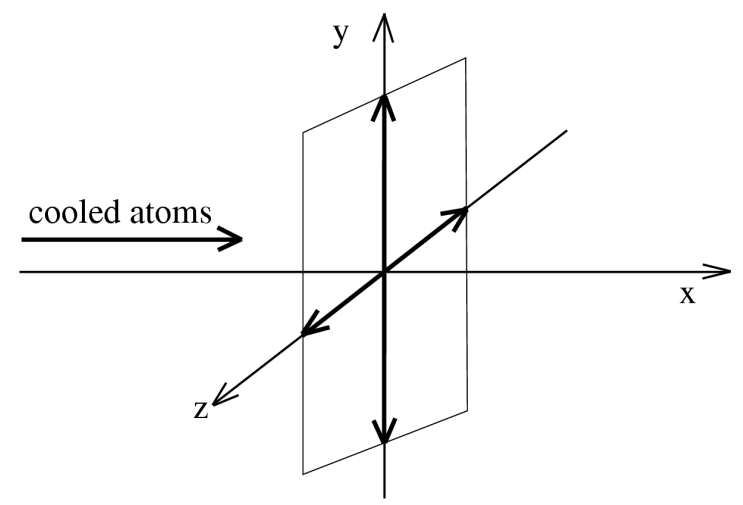

Fig. 1. Geometric structure of 2D control optical fields.

We can conclude from Eq. (10) that the atomic dynamics structure is simpler than for the mechanical pendulum, because two equations in (10) are independent. For this reason in the case of fast control we can apply the same scheme as for the $1 \mathrm{D}$ case, extending it into $2 \mathrm{D}$ to form periodical structures. Of course, applying different parameters of $u_{1}, u_{2}$, we can fabricate spatial distributions with different periods with respect to $y$ and $z$ directions. 

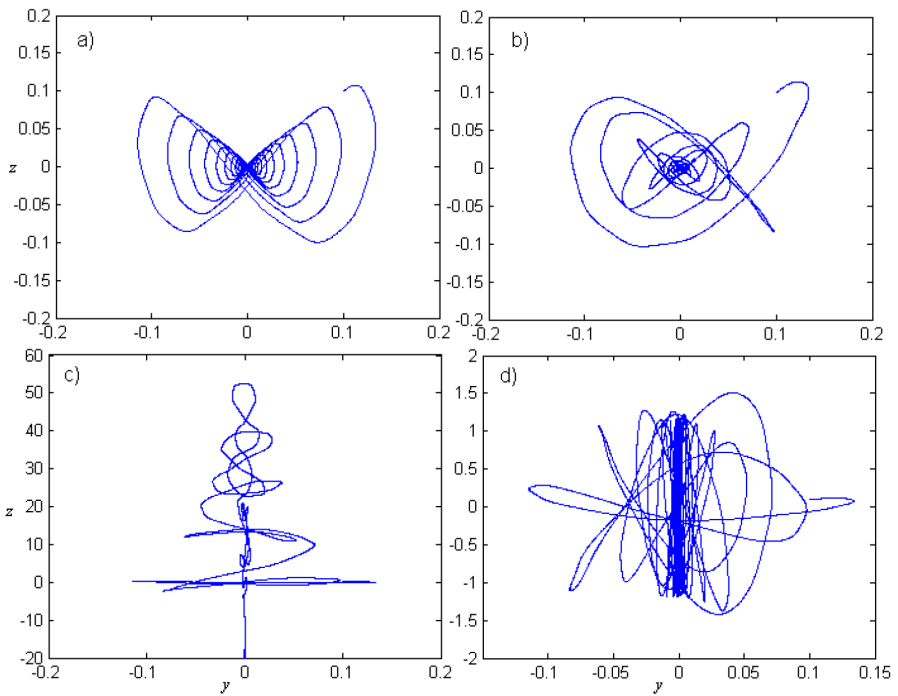

Fig. 2. $f=\sin , \varphi=0$.
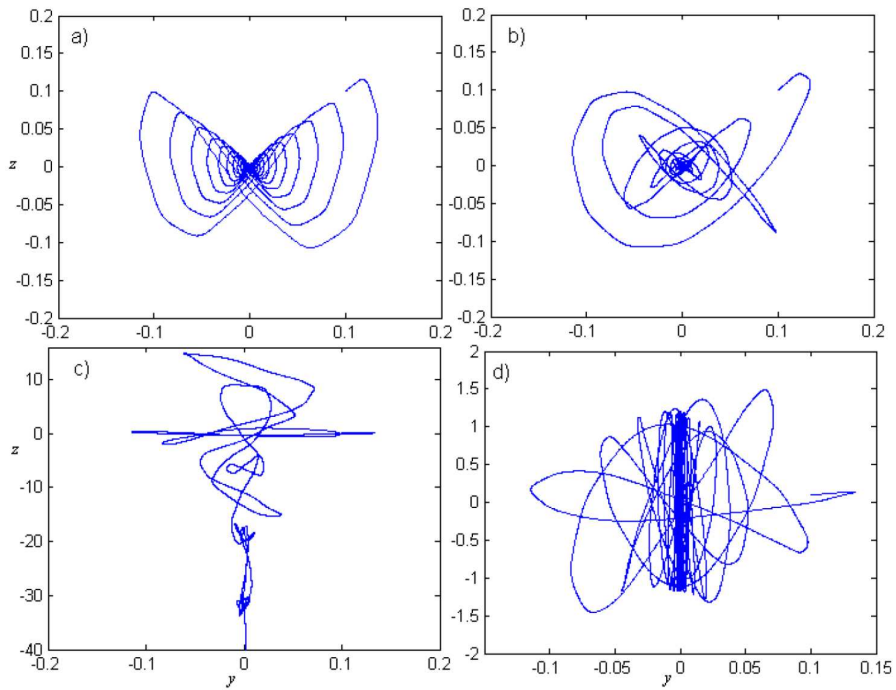

Fig. 3. $f=\sin , \varphi=30$.

Nevertheless, we should give a warning that in general our model reflects an averaged behavior with respect to the smooth coordinates $Y$ and $Z$. But another behavior can be observed for the non-averaged particle dynamics with different $\nu_{1}, \nu_{2}$, which are not necessary much greater than $\omega_{1} / \omega_{2}$. This warning can be especially important in $2 \mathrm{D}$ case, because we know from classical mechanics that the rationality or irrationality of the relations $\omega_{1} / \omega_{2}$ and $\nu_{1} / \nu_{2}$ can change the phase trajectories drastically. 

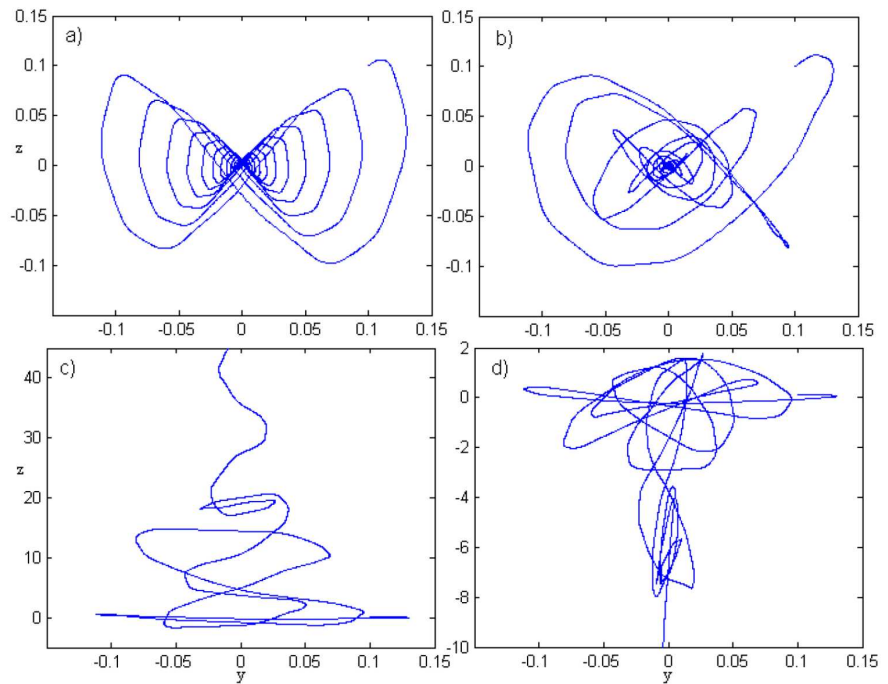

Fig. 4. $f=\operatorname{signum}(\sin ), \varphi=0$.

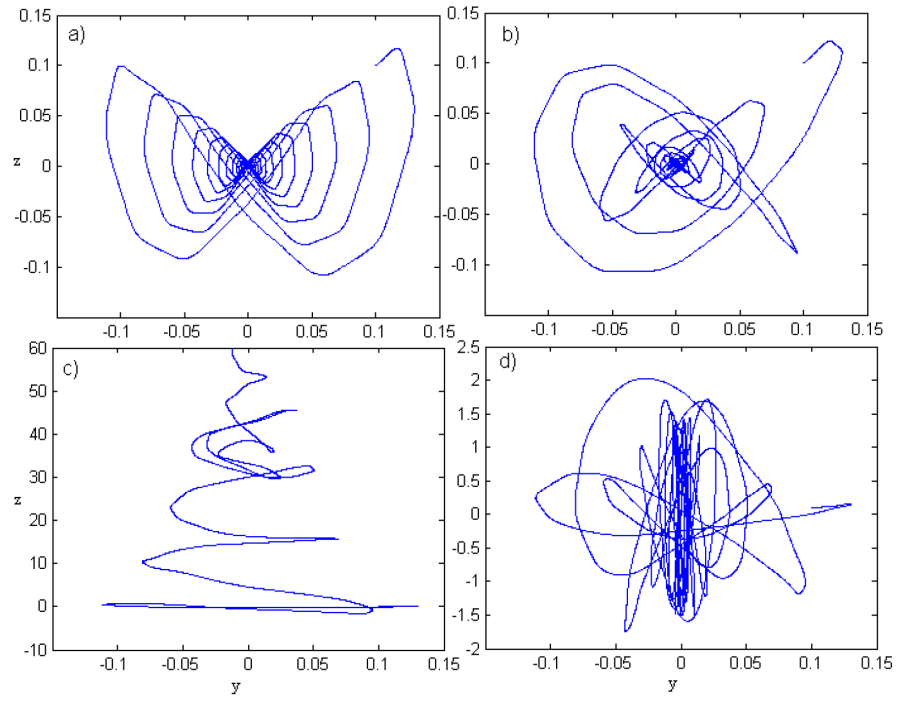

Fig. 5. $f=\operatorname{signum}(\sin ), \varphi=30$.

The detailed numerical investigation of the system behavior is not a subject of this paper. But to make the picture more clear, let us discuss here some simulations of single particle behavior under the dynamics (10).

As we saw in Sect. 2, the initial phase $\varphi$ is not sufficient for the averaged system. Let us put it to be 0 for $y$-control, and take it $\varphi$ for $z$-control. We have 4 different variants for focusing attractors at the points $(0,0,0,0),(0,0, \pi, 0)$, $(\pi, 0,0,0)$ and $(\pi, 0, \pi, 0)$ (they have no simple mechanical analogues anymore). 

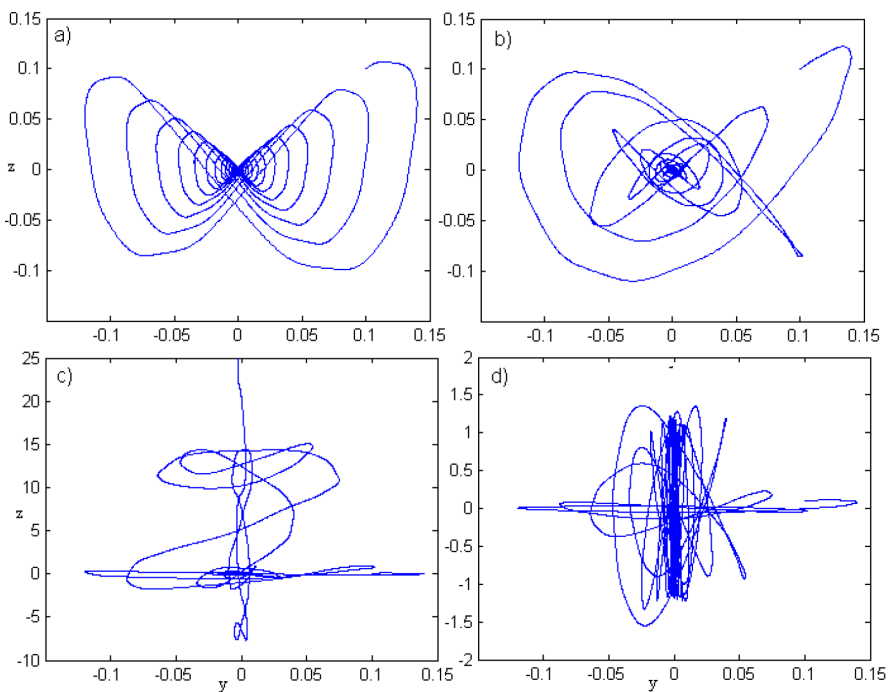

Fig. 6. $f=\cos , \varphi=0$.
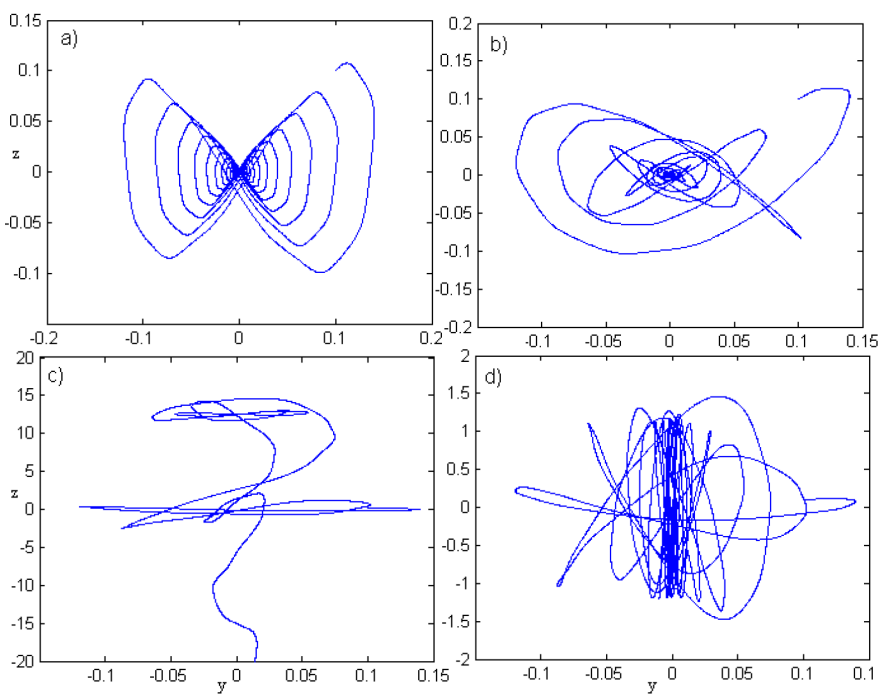

Fig. 7. $f=\cos , \varphi=30$.

In Figs. 2-7 we draw phase portraits (on $y z$-plane) of our model at the fixed point $(0,0,0,0)$ for different shapes of open-loop signals $u_{1,2}$. We put the same shape for both control signals, and apply the same amplitudes (these signals are much more simple to be created practically). We apply sin, signum of sin, and cos to demonstrate that for the fast control $\left(\nu_{1} \gg \omega_{1}\right.$ and $\left.\nu_{2} \gg \omega_{2}\right)$ the shape of control signal is not very sufficient. We also can observe relatively small difference 
for different meanings of the initial phase $\varphi$. What is really important, as we can expect, it is the relations of $\nu_{1}, \nu_{2}, \omega_{1}$ and $\omega_{2}$.

In Figs. 2-7 we plot 4 pictures: the left column corresponds to the rational relations of $\omega_{1} / \omega_{2}$, the right column - irrational $\omega_{1} / \omega_{2}$; the row above contains the rational relation of $\nu_{1} / \nu_{2}$, the row below - irrational. From the comparison we can conclude that the rational relation for $\omega_{1} / \omega_{2}$ is preferable, and for $\nu_{1} / \nu_{2}$ the rationality/irrationality is not very sufficient.

\section{Conclusions}

It is known very well that the methods of fast oscillating control of Kapitza's type are efficiently applicable for different systems of classical physics. In quantum mechanics the picture is different, and the difficulties come from the extending of mathematical language of classical control into non-classical areas. Nevertheless, in the case of quasi-classical models (like in this paper) the formulation of open-loop methods (with control fields depending only on time) is not problematic.

Our model of control for focusing of quantum neutral particles has some important features:

1. It can be very easily realized in experiment, because now there are no difficulties to construct different shapes of optical laser fields.

2. It allows to re-formulate it for a wide spectrum of open-loop and closed-loop control methods, i.e. for the cases of coordinate-dependent or independent $u_{1,2}$. The natural extension can be the detailed investigations of different shapes of control, not necessary harmonic.

3. We can change the type of attractor in the fixed points of our system by changing the parameters of control optical field.

\section{Acknowledgments}

We are grateful to Dr. Alexander Fradkov and Dr. Yuri Rozhdestvensky' for their useful remarks and productive discussion.

\section{Appendix. Phase portraits on $y z$ plane}

In Figs. 2-7 we construct the phase portraits of the system (10) on $y z$ plane. We draw the plots in the neighborhood of the fixed point $(0,0,0,0)$. Here

$u_{1}(t)=A_{1} f_{1}\left(\nu_{1} t\right) ; \quad u_{2}(t)=A_{2} f_{2}\left(\nu_{2} t+\varphi\right)$.

In every Figs. $2-7: \beta=0.1, A_{1}=A_{2}=1, f_{1}=f_{2} \equiv f$, the set of initial conditions: $y(0)=z(0)=0.1, \dot{y}(0)=\dot{z}(0)=0$; and

(a) $\omega_{1}=1, \omega_{2}=2, \nu_{1}=10, \nu_{2}=12$;

(b) $\omega_{1}=1, \omega_{2}=\sqrt{2}, \nu_{1}=10, \nu_{2}=12$;

(c) $\omega_{1}=1, \omega_{2}=2, \nu_{1}=10, \nu_{2}=\sqrt{10}$;

(d) $\omega_{1}=1, \omega_{2}=\sqrt{2}, \nu_{1}=10, \nu_{2}=\sqrt{10}$. 
In Figs. 2 and 3 we take the sin shape of the control signals, in Figs. 4 and $5-\operatorname{signum}(\sin )$, and in Figs. 6 and $7-\cos$. Thus, we put figures for the different shapes $f$ of the control signals and the different initial phase $\varphi$ (the phase is measured in grades).

We remind that in our model all the parameters are dimensionless.

\section{References}

[1] Atom Interferometry, Ed. P.R. Berman, Academic Press, New York 1997.

[2] A.G. Truscott, M.E.J. Friese, W.K. Hensinger, H.M. Wiseman, H. Rubinsztein-Dunlop, N.R. Heckenberg, Phys. Rev. Lett. 84, 4023 (2000).

[3] W.R. Anderson, C.C. Bradley, J.J. McClelland, R.J. Celotta, Phys. Rev. A 59, 2476 (1999).

[4] S.V. Borisenok, Yu.V. Rozhdestvensky, A.L. Fradkov, B.R. Andrievsky, B.G. Matisov, in: Proc. 2003 Int. Conf. "Physics and Control", Russian Academy of Sciences, St. Petersburg 2003, p. 906.

[5] A.L. Fradkov, A.Yu. Pogromsky, Introduction to Control of Oscillations and Chaos, World Scientific, Singapore 1999.

[6] P.L. Kapitza, J. Exp. Theor. Phys. 21, 588 (1951).

[7] L.D. Landau, E.M. Lifshitz, Mechanics, Pergamon Press, Oxford 1960.

[8] R. Clark Robinson, An Introduction to Dynamical Systems: Continuous and Discrete, Pearson Prentice Hall, Singapore 2004. 